\title{
The Fairing of Ship Lines on a High-Speed Computer
}

\section{By Feodor Theilheimer and William Starkweather}

\begin{abstract}
Methods for using a digital high-speed computer to determine ship lines are presented. It is assumed that the offsets of a small number of points were taken from a preliminary design, and that it is desired to compute the offsets of an arbitrarily large number of points on the ship's surface. Procedures for using a computer for the solution of this problem are described. Special emphasis is placed on the detection, by a computational criterion, of unwanted fluctuations and the correction of such fluctuations.if they should occur. The method also includes a special procedure which takes care that those portions which are straight in the preliminary design remain straight in the final form. Illustrative examples of the methods are discussed.
\end{abstract}

1. Introduction. Before the actual construction of a ship can begin, considerable time and effort has to be spent in the drawing of the ship lines. The purely graphical methods of determining ship lines are very tedious and time-consuming. Therefore, the problem of implementing the graphical methods by analytic procedures has been studied for a considerable time.

One of the most important earlier contributions is due to Admiral David W. Taylor [1]. An extensive history and bibliography of the problem is given in Volume II. of "Hydrodynamics in Ship Design," by Captain Harold E. Saunders [2]. Among the more recent publications are papers by W. H. Rösingh and J. Berghius [3], P. C. Pien [4], and J. E. Kerwin [5].

The analytical approach to the ship line problem has become particularly attractive since high-speed computers became available. This offers an opportunity to eliminate much of the drudgery inherent in the graphical method.

When ship lines are found by an analytic method, they may possess unwanted fluctuations. It is, therefore, desirable to have an analytic criterion which permits us to determine whether or not a line is free of unwanted fluctuations. This paper furnishes such a criterion and gives a method of finding lines without such fluctuations.

In Section 2 a method, which essentially amounts to an interpolation, is developed for finding a ship surface which passes exactly through a set of points given in a preliminary design.

In Section 3 this method is modified to a smoothing procedure which yields ship lines that are free of unwanted fluctuations but which may no longer pass exactly through the given points.

Section 4 deals with the special case where the preliminary design contains straight line portions, and a method which reproduces these lines as straight lines is developed.

Received May 15, 1961. This paper is reprinted from David Taylor Model Basin Report 1474, January 1961. 


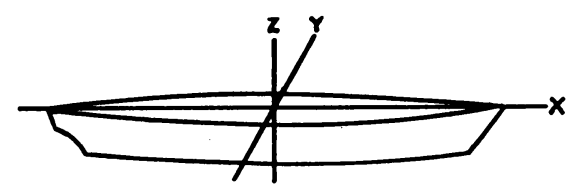

Fig. 1.-The Coordinate Axes

Section 5 describes applications of the various procedures for finding ship lines by a high-speed computer.

2. Interpolation Method. The coordinate system is taken as indicated in Figure 1; the $x$-axis is longitudinal, the $z$-axis is vertical, and the $y$-axis is perpendicular to both the $x$ - and $z$-axes.

The determination of ship lines by analytic methods is considered equivalent to finding a function of two variables

$$
y=F(x, z)
$$

so that for every fixed value of $z$ we have $y$ as a function of $x$ along a waterline, which permits us to compute an arbitrarily large number of half-breadths on that waterline. Likewise, a fixed value of $x$ would yield an arbitrarily large number of half-breadths along a section.

We assume that the ship for which we are to determine $y=F(x, z)$ is described to us by a table of offsets taken from a preliminary design.

Let the offsets be given at $N+1$ points on each of $K+1$ waterlines. Then we will develop a procedure which yields $y=F(x, z)$ and thus gives an arbitrarily large number of offsets instead of the given $(N+1)(K+1)$ points. The existing program for the computing machine is set up for handling cases where $N$ and $K$ can be as large as 24 , but, in the cases actually treated, $N$ and, particularly, $K$ were much smaller.

To find a function $y=F(x, z)$ we first determine a function $y=f(x)$ which corresponds to a single waterline. The finding of such a function $y=f(x)$ will turn out to be an essential part of the determination of the surface $y=F(x, z)$.

We assume that the entire waterline is divided into $N$, not necessarily equal, intervals and that the $N+1$ points $\left(x_{0}, y_{0}\right), \cdots,\left(x_{N}, y_{N}\right)$ are given. We now consider the problem of finding a curve which passes exactly through these points. This interpolation problem can be attacked only after we make a choice as to the type of curve that should represent a waterline.

We shall insist that the function be continuous and have continuous slope and curvature or, what amounts to the same, have continuous first and second derivatives.

A simple type of curve that satisfies these conditions is the following:

Let the curve consist of a number of segments, each segment being represented by a cubic. These cubics are joined in such a way that at the juncture points the function, its first derivative, and its second derivative are continuous.

Curves of this type arise in the theory of small deflections of thin beams which are simply supported at a finite number of points. To some extent a batten or spline held in place by so-called ducks, as it is used in the drawing of ship lines, 
can be approximated by a thin beam simply supported at a finite number of points. The analogy between a spline and a thin beam gave rise to the name "spline curve" for the type of curve consisting of cubic segments described here.

We have chosen spline curves to represent ship lines. The explicit formula for a spline curve is greatly simplified if we introduce the following notation:

$$
\begin{aligned}
(x-a)_{+}{ }^{3} & =0 & & \text { for } x \leqq a \\
& =(x-a)^{3} & & \text { for } x \geqq a .
\end{aligned}
$$

Equation (2) can also be written in the form:

$$
(x-a)_{+}{ }^{3}=\frac{1}{2}\left[(x-a)^{3}+|x-a|^{3}\right] \text {. }
$$

With the aid of this notation, a spline curve which has discontinuities of the third derivative at the points $x_{1}, \cdots, x_{N-1}$ can be written as

$$
\begin{aligned}
f(x)=y=a+b x+c x^{2} & +A_{0} x^{3} \\
& +A_{1}\left(x-x_{1}\right)_{+}{ }^{3}+\cdots+A_{N-1}\left(x-x_{N-1}\right)_{+}{ }^{3} .
\end{aligned}
$$

It is clear that each new term

$$
A_{n}\left(x-x_{n}\right)_{+}^{3}, \quad n=1, \cdots, N-1
$$

introduces a discontinuity of the third derivative at $x=x_{n}$, the magnitude of the jump in the third derivative being equal to $6 A_{n}$, whereas the continuity of the function, its first derivative, and its second derivative remain undisturbed.

In each of the $N$ intervals

$$
x_{n-1}<x<x_{n}, \quad n=1,2, \cdots, N
$$

formula (4) sums to a single cubic. In individual cases any of the four terms of such a cubic may drop out in the summation, and, in particular, it is possible that the cubic may reduce to a straight line. This shows that the spline curve lends itself to the representation of curves which contain straight portions as, for instance, waterlines on ships with parallel middlebody.

We shall now develop a procedure for determining the coefficients of a spline curve which passes exactly through the $N+1$ points

$$
\left(x_{0}, y_{0}\right),\left(x_{1}, y_{1}\right), \cdots,\left(x_{N}, y_{N}\right) \text {. }
$$

We choose as points of discontinuity of the third derivative the $N-1$ inner points

$$
\left(x_{1}, y_{1}\right), \cdots,\left(x_{N-1}, y_{N-1}\right)
$$

of the given set of points. The spline curve then appears in the form of equation (4) which has $N+3$ coefficients. For the determination of these coefficients we have $N+1$ equations; namely, the conditions that the curve should pass through the $N+1$ given points, $y_{n}=f\left(x_{n}\right)$ for $n=0,1, \cdots, N$, which means that we have two more unknowns than equations.

To get a clearer picture of the role of these two degrees of freedom, we rewrite $f(x)$ of equation (4) in the form

$$
f(x)=f_{0}(x)+p f_{1}(x)+q f_{2}(x)
$$


where $f_{0}, f_{1}$, and $f_{2}$ are to satisfy the following conditions:

$$
\begin{array}{llll}
f_{0}\left(x_{n}\right)=y_{n} & f_{1}\left(x_{n}\right)=0 & f_{2}\left(x_{n}\right)=0, & n=0,1, \cdots, N \\
f_{0}{ }^{\prime}\left(x_{0}\right)=0 & f_{1}{ }^{\prime}\left(x_{0}\right)=1 & f_{2}{ }^{\prime}\left(x_{0}\right)=0 \\
f_{0}{ }^{\prime \prime}\left(x_{0}\right)=0 & f_{1}^{\prime \prime}\left(x_{0}\right)=0 & f_{2}^{\prime \prime}\left(x_{0}\right)=2 .
\end{array}
$$

Each one of the functions $f_{0}, f_{1}, f_{2}$ is now determined by $N+3$ conditions. The requirements at $x_{0}$ are clearly satisfied if we write:

$$
\begin{aligned}
& \begin{array}{l}
f_{0}(x)=y_{0}+B_{0}\left(x-x_{0}\right)^{3} \\
+
\end{array} \\
&+B_{1}\left(x-x_{1}\right)_{+}{ }^{3}+\cdots+B_{N-1}\left(x-x_{N-1}\right)_{+}{ }^{3} \\
& f_{1}(x)=x-x_{0}+C_{0}(x-\left.x_{0}\right)^{3} \\
&+C_{1}\left(x-x_{1}\right)_{+}{ }^{3}+\cdots+C_{N-1}\left(x-x_{N-1}\right)_{+}{ }^{3} \\
& f_{2}(x)=\left(x-x_{0}\right)^{2}+D_{0}\left(x-x_{0}\right)^{3} \\
&+
\end{aligned}
$$

The $B_{n}, C_{n}$, and $D_{n}, n=0, \cdots, N-1$, can be found by utilizing the equations $f_{0}\left(x_{n}\right)=y_{n}, f_{1}\left(x_{n}\right)=0, f_{2}\left(x_{n}\right)=0, n=1, \cdots, N$. To find these coefficients one has three systems each of $N$ equations in $N$ unknowns, which are obtained by substituting the values of $x_{1}, x_{2}, \cdots, x_{N}$ in place of $x$ in the three equations of (7), and by utilizing the first line of equation (6). The coefficients $B_{n}, C_{n}$, and $D_{n}$ can be found without solving the systems of equations in the usual manner. Substituting $x=x_{1}$ immediately yields $B_{0}, C_{0}$, and $D_{0}$, and if we put $x=x_{n}$, we find $B_{n}$ in terms of $B_{0}, B_{1}, \cdots, B_{n-1}$ and similarly, $C_{n}$ and $D_{n}$ in terms of the preceding $C$ 's and $D$ 's, respectively.

After the functions $f_{0}, f_{1}$, and $f_{2}$ are thus determined, the parameters $p$ and $q$ of equation (5) must be found. Among the curves

$$
y=f_{0}+p f_{1}+q f_{2}
$$

which all pass through the $N+1$ points $\left(x_{0}, y_{0}\right), \cdots,\left(x_{N}, y_{N}\right)$, we have to choose one which is by some criterion most desirable.

Between two spline curves, we will consider as more desirable the one which has smaller jumps of the third derivative, or more precisely, the one where the sum of the squares of the jumps of the third derivative is less. Going back to thin beam theory, we note that the reaction force at a point of support is proportional to the jump in the third derivative. The desired spline curve is, therefore, the one for which

$$
\sum_{n=1}^{N-1}\left(A_{n}\right)^{2}
$$

becomes a minimum, since $6 A_{n}$ is the jump in the third derivative at $x_{n}$ for the function $f(x)$ of equation (4).

From equations (5) and (7) we see that

$$
A_{n}=B_{n}+p C_{n}+q D_{n}
$$


and therefore

$$
\sum_{n=1}^{N-1}\left(B_{n}+p C_{n}+q D_{n}\right)^{2}
$$

has to become a minimum.

This leads to the following two linear equations in $p$ and $q$

$$
\begin{aligned}
& p \Sigma C_{n}{ }^{2}+q \Sigma C_{n} D_{n}=-\Sigma B_{n} C_{n} \\
& p \Sigma C_{n} D_{n}+q \Sigma D_{n}{ }^{2}=-\Sigma B_{n} D_{n}
\end{aligned}
$$

where we always sum in $n$ from 1 to $N-1$.

By this method a spline curve which passes through $N+1$ given points is found in the form:

$$
f(x)=y_{0}+p\left(x-x_{0}\right)+q\left(x-x_{0}\right)^{2}+A_{0}\left(x-x_{0}\right)^{3}+\sum_{n=1}^{N-1} A_{n}\left(x-x_{n}\right)_{+}^{3} .
$$

By expanding the powers of $\left(x-x_{0}\right)$, this can be brought into the form

$$
f(x)=a+b x+c x^{2}+A_{0} x^{3}+\sum_{n=1}^{N-1} A_{n}\left(x-x_{n}\right)_{+}{ }^{3} .
$$

We have thus developed a procedure for finding a spline curve which represents a waterline that passes through $N+1$ given points. If we are given $N+1$ points on $K+1$ waterlines, we can therefore find $K+1$ functions, $f_{k}(x), k=0, \cdots, K$, expressing the $K+1$ waterlines. Our next step will then be to find $y=F(x, z)$ so that

$$
F\left(x, z_{k}\right)=f_{k}(x)
$$

where $z_{k}$ is the $z$ coordinate of the $k$ th waterline.

Each one of the functions $f_{k}(x)$ appears in the form of $f(x)$ as given in equation (4) where the coefficients $a, b, c, A_{0}, A_{1}, \cdots, A_{N-1}$ are generally different for each $f_{k}(x)$. Each one of these coefficients, say for example, $a$ is now given for $z=z_{0}$, $z=z_{1}, \cdots, z=z_{\boldsymbol{K}}$. We can therefore ask for a function $a(z)$ for which we are given values at $z_{0}, z_{1}, \cdots, z_{K}$. This is exactly the same problem that confronted us when we were given $N+1$ points on a waterline and found $f(x)$.

By using the same procedure which gave us $f(x)$ we can now find $a(z)$ as a spline function of $z$. In the same manner $b(z), c(z), A_{0}(z), \cdots, A_{N-1}(z)$ can be found. This gives the expression for the whole surface

$$
\begin{aligned}
F(x, z)=a(z) & +b(z) \cdot x+c(z) \cdot x^{2}+A_{0}(z) \cdot x^{3} \\
& +A_{1}(z) \cdot\left(x-x_{1}\right)_{+}{ }^{3}+\cdots+A_{N-1}(z) \cdot\left(x-x_{N-1}\right)_{+}{ }^{3} \cdot
\end{aligned}
$$

Each one of the $N+3$ coefficient functions $a(z), b(z), c(z), A_{0}(z), A_{1}(z), \cdots$, $A_{N-1}(z)$ is itself a sum of $K+3$ terms.

In this derivation we worked under the assumption that the abscissas $x_{n}$, $n=1, \cdots, N-1$ do not change from waterline to waterline. In general, we assume that the data given originally will satisfy this requirement. However, we permit $x_{0}$ and $x_{N}$, which do not appear explicitly in the formula for the spline curve, 
to vary from waterline to waterline. This is very advantageous since, in general, waterlines on different levels do not have the same $x_{0}$ and $x_{N}$.

In order to apply our procedure as it stands, it is necessary that $x_{1}, \cdots, x_{N-1}$ do not change from waterline to waterline. However, a simple modification of our procedure will permit us to handle the case where some waterlines do not conform to this restriction.

If some waterlines have offsets given at $x_{0}, x_{1}, \cdots, x_{N}$, whereas one waterline has offsets given at $x_{0}{ }^{*}, x_{1}{ }^{*}, \cdots, x_{N}{ }^{*}$, then we can find a function which expresses this waterline in a form analogous to equation (13) by means of a function

$$
f(x)=a+b x+c x^{2}+A_{0} x^{3}+A_{1}\left(x-x_{1}{ }^{*}\right)_{+}{ }^{3}+\cdots+A_{N-1}\left(x-x_{N-1}^{*}\right)_{+}{ }^{3} .
$$

From this function we can find $f\left(x_{1}\right), f\left(x_{2}\right), \cdots, f\left(x_{N-1}\right)$ which can serve as ordinates of modified data which go with the abscissas $x_{1}, \cdots, x_{N-1}$. Such a modification can also be used if one line contains fewer points than the other lines.

The derivation of $F(x, z)$ described here shows immediately that the originally given points on each waterline do not have to have equidistant abscissas nor do the waterlines have to be equally spaced.

A program to compute the coefficients of the function $y=F(x, z)$ was coded for the Remington Rand high-speed computer UNIVAC. Further computer programs were then developed to evaluate this function and to print out in tabular form the offsets along any portion of any waterline if $z$ is given and the first desired value of $x$, an increment of $x$, and the number of $x$ values desired are entered into the machine. In similar fashion, any portion of any transverse section can be tabulated.

These various machine programs were later recoded for the IBM 704 computing system.

It is clear that the determination of the coefficients for the function $y=F(x, z)$ constitutes the main computing effort. The evaluation of the function and the printing of the results for any number of points requires very little extra time.

3. Smoothing Method. The procedure described thus far usually gives very satisfactory results, but in some cases it turned out that the computed ship lines showed unwanted fluctuations which could be recognized by plotting the lines. There arises a need for a criterion which will permit the computer to determine whether or not a line is free of such unwanted fluctuations.

Clearly, a curve that has no inflection points would be free of all fluctuations. We cannot require, however, that ship lines have no inflection points, because there are well-designed ship lines that do have inflection points. What we want to avoid are extraneous inflection points. We therefore have to determine where the given data indicate the presence or absence of an inflection point.

This can be done by studying three successive points $P_{n-1}, P_{n}, P_{n+1}$ with the coordinates $\left(x_{n-1}, y_{n-1}\right),\left(x_{n}, y_{n}\right),\left(x_{n+1}, y_{n+1}\right)$. If the three points are plotted and $P_{n-1}$ and $P_{n+1}$ are joined by a straight line, it can readily be seen whether the curve in the vicinity of $P_{n}$ is concave upward or downward. This graphical method can be easily translated into analytical form by introducing the second difference, which here shall be called $r_{n}$.

Then the sign of the second difference will indicate whether the curve is to be 
concave upward or downward at $P_{n}$. A positive second difference indicates concave upward, a negative second difference indicates concave downward.

The formula for the second difference becomes particularly simple in the case of equally spaced abscissas:

$$
\left(x_{n+1}-x_{n}\right)=\left(x_{n}-x_{n-1}\right)=h .
$$

Then the second difference becomes

$$
r_{n}=\frac{y_{n+1}-2 y_{n}+y_{n-1}}{h^{2}} .
$$

In the case where the abscissas are not equally spaced, formula (15) is to be replaced by the general formula

$$
r_{n}=\frac{2}{x_{n+1}-x_{n-1}}\left(\frac{y_{n+1}-y_{n}}{x_{n+1}-x_{n}}-\frac{y_{n}-y_{n-1}}{x_{n}-x_{n-1}}\right) .
$$

If we are given the $N+1$ points

$$
\left(x_{0}, y_{0}\right), \cdots,\left(x_{N}, y_{N}\right)
$$

we can readily determine $N-1$ second differences

$$
r_{1}, \cdots, r_{N-1} \text {. }
$$

If $r_{n}$ and $r_{n+1}$ have the same sign, we can consider this an indication that there should not be an inflection point between $x_{n}$ and $x_{n+1}$. If they have different signs, the given data suggest an inflection point between $x_{n}$ and $x_{n+1}$. We are now in a position to determine whether or not a curve $y=f(x)$ has unwanted fluctuations. Only if the second derivative $f^{\prime \prime}\left(x_{n}\right)$ and the second difference $r_{n}$ have the same sign can we be sure that the curve $y=f(x)$ is free of unwanted fluctuations.

Here it is understood that a function whose second derivatives at $x_{n}$ and $x_{n+1}$ have the same sign has no inflection points between $x_{n}$ and $x_{n+1}$. This, however, will not be true for every function; for instance, it will not be true for a polynomial of degree higher than three. Such a function can have an even number of inflection points between two successive points at which the second derivatives have equal signs. However, if we deal with spline curves, $x_{n}$ and $x_{n+1}$ are joined by a cubic, which can possess not more than one single inflection point. By the same reasoning, we find that if in the case of a spline curve, $f^{\prime \prime}\left(x_{n}\right)$ and $f^{\prime \prime}\left(x_{n+1}\right)$ have different signs, there will be precisely one inflection point in the interval $x_{n}$ to $x_{n+1}$, and not an arbitrary odd number as it might be for the general curve.

For illustration, we have discussed the comparison of the second differences of the data with the second derivatives of the computed curve only in the case where the curve has been a waterline, and we have, therefore, been finding a function of $x$. Exactly the same test can, of course, be carried out when we are finding a function of $z$.

The test of whether or not a given curve has unwanted fluctuations can be carried out very easily by the computing machine for either waterlines or sections, and the program can be written so that the computing machine prepares a listing of the points where the curve fails the test. It may occasionally happen that the 
discrepancies are of a trivial nature, but if they are not, a method must be devised to compute new lines which are free of unwanted fluctuations.

In trying to free ourselves from unwanted fluctuations we think of a smoothing procedure which relaxes the requirement that the computed curve should pass exactly through the given points.

The method of least squares immediately suggests itself. If, however, a leastsquares method is applied, one finds that in comparatively simple cases one is led to unwanted fluctuations. The fact that a least-squares fit may yield fluctuations can be ascribed to the following consideration.

A least-squares fit minimizes the sum of the squares of the differences between the given and computed values but imposes no constraint to minimize fluctuations. If we want to free ourselves of fluctuations, we have to take care that the second difference and the second derivative have the same sign in each case. This can be attempted by minimizing the sum of the squares of the discrepancies between the second differences and the second derivatives.

Such a minimization is essentially implicit in the interpolation procedure described by equations (4) through (10). There we minimize $\Sigma\left(A_{n}\right)^{2}$. It can be shown that

$$
A_{n}=\frac{1}{h}\left[r_{n}-f^{\prime \prime}\left(x_{n}\right)\right]
$$

in the case where the abscissas are equally spaced with the spacing $h$.

Let $x_{n}$ be a point where the third derivative of a spline curve undergoes a jump

$$
6 A_{n}=f^{\prime \prime \prime}\left(x_{n+}\right)-f^{\prime \prime \prime}\left(x_{n-}\right)
$$

then

$$
\begin{aligned}
& f\left(x_{n}+h\right)=f\left(x_{n}\right)+h f^{\prime}\left(x_{n}\right)+\frac{h^{2}}{2} f^{\prime \prime}\left(x_{n}\right)+\frac{h^{3}}{6} f^{\prime \prime \prime}\left(x_{n+}\right) \\
& f\left(x_{n}-h\right)=f\left(x_{n}\right)-h f^{\prime}\left(x_{n}\right)+\frac{h^{2}}{2} f^{\prime \prime}\left(x_{n}\right)-\frac{h^{3}}{6} f^{\prime \prime \prime}\left(x_{n-}\right) .
\end{aligned}
$$

If we add equations (19) and (20) and utilize equations (15) and (18), we get equation (17).

Therefore, when we used the interpolation method described by equations (4) through (10), we found among all possible spline curves passing exactly through the $N+1$ given points the one for which, in the case of equally spaced points, the discrepancies between the second differences and the second derivatives were least.

Even if we minimize the discrepancies between the second differences and the second derivatives but insist that the curve pass exactly through the given points, we will often get unwanted fluctuations. To free ourselves from these fluctuations, we have to relax the requirement that the computed curve pass exactly through the given points.

If we no longer insist that the curve pass exactly through the given points, we do not require that $f\left(x_{n}\right)$ be equal to $y_{n}$, but we still want to make

$$
\sum_{n=0}^{N}\left[f\left(x_{n}\right)-y_{n}\right]^{2}
$$


small. To avoid extraneous fluctuations we also want to make $\sum_{n=1}^{N-1}\left[f^{\prime \prime}\left(x_{n}\right)--r_{n}\right]^{2}$ small. We therefore minimize a linear combination of the two sums; namely,

$$
\sum_{n=0}^{N}\left[f\left(x_{n}\right)-y_{n}\right]^{2}+s \sum_{n=1}^{N-1}\left[f^{\prime \prime}\left(x_{n}\right)-r_{n}\right]^{2} .
$$

The use of the parameter $s$ enables us to emphasize either the first or the second sum. By choosing $s$ small we put more stress on minimizing the first sum, which makes the curve pass more closely to the given points. By choosing $s$ large we put more stress on minimizing the second sum, which makes the discrepancy between the second derivative and the second difference small. This tends to make them agree in sign, and thus tends to eliminate unwanted fluctuations.

If we study the case of $s=0$ we minimize only the first sum. This sum has a zero minimum, since each summand can be made zero individually by solving the $N+1$ linearly independent equations, $f\left(x_{n}\right)=y_{n}, n=0,1, \cdots, N$, for the $N+3$ unknown coefficients, $a, b, c, A_{0}, \cdots, A_{N-1}$. Therefore $s=0$ leads to a curve passing exactly through the given points. This curve, however, would not be uniquely determined but would depend on two parameters just as in equation (5).

A similar situation would prevail if we were to minimize only the second sum in formula (21), which would be equivalent to letting $s$ become infinite. Then we would also get a zero minimum, and we would achieve perfect agreement between the second derivatives and the second differences. For sufficiently large $s$ we can therefore make the second sum as small as we please and obtain agreement in sign between second derivatives and second differences.

Thus we have found a method for computing a curve which is suggested by $N+1$ points. We first minimize expression (21) with a small $s$ and then test to see if there are any unwanted fluctuations. If there are some, we increase $s$ until the curve is satisfactory. When all waterlines have been fitted with satisfactory curves, we can then fit like coefficients into functions of $z$ by utilizing formula (21) again, and thereby find the expression for the ship's surface $y=F(x, z)$.

Although one may choose different $s$ values for different lines and even for different points on a given line, most of the work has been done primarily by using the same value of $s$ throughout for any given run on the computer.

4. Lines With Straight Portions. One of the reasons for choosing spline curves as typical ship lines was the fact that ship lines often contain straight portions. A polynomial or any other analytic function cannot contain straight as well as curved portions. Although spline curves can contain straight portions as well as curved ones, the methods developed thus far do not contain any special provisions which will ensure that straight portions will be reproduced as straight portions.

It is felt that every effort should be made to reproduce exactly those portions of the preliminary design which are clearly intended to be straight.

First, we will treat the case where the straight portion is at the beginning of the line.

Suppose that of the $N+1$ points

$$
\left(x_{n}, y_{n}\right), \quad n=0, \cdots, N
$$

the first $L+1$ points lie on a straight line of slope $a$. 
Then

$$
y_{n}=y_{0}+a\left(x_{n}-x_{0}\right) \quad \text { for } n=0, \cdots, L
$$

and the desired function can be written

$$
f(x)=y_{0}+a\left(x-x_{0}\right)+\sum_{n=\mathrm{L}}^{N-1} A_{n}\left(x-x_{n}\right)_{+}{ }^{3} .
$$

The $N-L$ unknown coefficients $A_{n}, n=L, \cdots, N-1$ could be found by solving the $N-L$ equations:

$$
\begin{gathered}
f\left(x_{L+1}\right)=y_{L+1}=y_{0}+a\left(x_{L+1}-x_{0}\right)+A_{L}\left(x_{L+1}-x_{L}\right)^{3} \\
\begin{array}{r}
f\left(x_{L+2}\right)=y_{L+2}=y_{0}+a\left(x_{L+2}-x_{0}\right) \\
+A_{L}\left(x_{L+2}-x_{L}\right)^{3}+A_{L+1}\left(x_{L+2}-x_{L+1}\right)^{3} \\
\cdots \\
f\left(x_{N}\right)=y_{N}=y_{0}+a\left(x_{N}-x_{0}\right)+A_{L}\left(x_{N}-x_{L}\right) \\
+A_{L+1}\left(x_{N}-x_{L+1}\right)^{3}+\cdots+A_{N-1}\left(x_{N}-x_{N-1}\right)^{3} .
\end{array}
\end{gathered}
$$

After having done this we would have to apply the previously derived criterion to find out whether or not there were any fluctuations. If there were unwanted fluctuations, we would then minimize the expression

$$
\sum_{n=\mathrm{L}+1}^{N}\left[f\left(x_{n}\right)-y_{n}\right]^{2}+s \sum_{n=\mathrm{L}+1}^{N-1}\left[f^{\prime \prime}\left(x_{n}\right)-r_{n}\right]^{2} .
$$

As before, a sufficiently large $s$ will ensure that the sign of the second derivative $f^{\prime \prime}\left(x_{n}\right)$ of the computed curve will agree with the sign of the second difference $r_{n}$ of the given data at each $x_{n}$.

If a ship line consists of a curved part followed by a straight part of slope $b$; that is, if, of the $N+1$ points,

$$
\left(x_{n}, y_{n}\right) \text {, }
$$

$$
n=0, \cdots, N
$$

the last $L+1$ points lie on a straight line, then

$$
y_{n}=y_{N}-b\left(x_{N}-x_{n}\right)
$$

for $n=N, N-1, \cdots, N-L$.

The expression for the ship line is then

$$
f(x)=y_{N}-b\left(x_{N}-x\right)+\sum_{n=N-L}^{1} A_{n}{ }^{*}\left(x_{n}-x\right)_{+}{ }^{3}
$$

where $\left(x_{n}-x\right)_{+}{ }^{3}=0$ for $\left(x_{n}-x\right)<0$.

The $A_{n}{ }^{*}$ can be determined by a procedure analogous to the one used in equations (24) or (25). The expression for a line thus determined will contain terms of the form $A_{n}^{*}\left(x_{n}-x\right)_{+}^{3}$, whereas we usually write lines with terms of the form $A_{n}\left(x-x_{n}\right)_{+}^{3}$.

The two forms are related to each other in the following way

$$
\left(x-x_{n}\right)_{+}{ }^{3}-\left(x_{n}-x\right)_{+}{ }^{3}=\left(x-x_{n}\right)^{3} \text {. }
$$


That this is a valid relationship becomes evident when we realize that for $x$ less than $x_{n}$, the first term on the left-hand side of equation (28) becomes zero, whereas the second term vanishes for $x$ greater than $x_{n}$.

Therefore, equation (27) can be modified in the following way:

$$
\begin{aligned}
f(x) & =y_{N}-b\left(x_{N}-x\right)+\sum_{n=N-\mathrm{L}}^{1} A_{n}{ }^{*}\left(x_{n}-x\right)_{+}{ }^{3} \\
& =\left[y_{N}-b\left(x_{N}-x\right)-\sum_{n=N-\mathrm{L}}^{1} A_{n}{ }^{*}\left(x-x_{n}\right)^{3}\right]+\sum_{n=N-\mathrm{L}}^{1} A_{n}{ }^{*}\left(x-x_{n}\right)_{+}{ }^{3} \cdot
\end{aligned}
$$

The terms inside the brackets can be arranged as a single cubic.

We have thus far treated the cases where a straight portion is followed by a curved portion and where a curved portion is followed by a straight portion. There is one more possibility; namely, that a line has a straight portion followed by a curved portion followed by a straight portion. If there are one or possibly more straight portions in the interior of a line, then we can subdivide each of the inner straight portions and treat the thus achieved cases individually.

The case of a curved portion between two straight portions leads to the problem of finding a spline curve for which the ordinates, the slopes, and the second derivatives of the two end points are given. In particular, the second derivatives are zero. Since six conditions are imposed, the spline curve must have at least six coefficients, which means that it must consist of at least three cubic arcs.

We first consider the case where there are two points given between the end points of the straight portions. Let $x_{L}$ be the abscissa of the end point of one of the straight lines and $x_{L+2}$ be the initial abscissa of the next straight line.

If

$$
\begin{array}{rlrl}
f\left(x_{L}\right) & =A & f\left(x_{L+3}\right) & =B \\
f^{\prime}\left(x_{L}\right) & =a & f^{\prime}\left(x_{L+3}\right) & =b \\
f^{\prime \prime}\left(x_{L}\right) & =0 & f^{\prime \prime}\left(x_{L+3}\right) & =0
\end{array}
$$

then

$$
f(x)=A+a\left(x-x_{L}\right)+\sum_{n=\mathrm{L}}^{\mathrm{L}+3} A_{n}\left(x-x_{n}\right)_{+}{ }^{3}
$$

which immediately satisfies the conditions at $x_{L}$.

The conditions at $x_{L+3}$ yield three linear equations in the three unknowns $A_{L}$, $A_{L+1}$, and $A_{L+2}$. The determinant of this system is recognized to be different from zero because it contains three rows which are the first, second, and third powers of $\left(x_{L+3}-x_{n}\right), \quad n=L, L+1, L+2$.

The coefficient $A_{L+3}$ is determined by the requirement that for $x>x_{L+3}$ the curve should be a straight line; namely, $f(x)=B+b\left(x-x_{L+3}\right)$.

The solution of this problem can be given explicitly for the case of equidistant abscissas $x_{L}, x_{L}+h, x_{L}+2 h, x_{L}+3 h$. To simplify the necessary formulas, we look at it as a combination of three elementary cases.

In the first case, if $f\left(x_{L}\right)=A$ and $f\left(x_{L}+3 h\right)=B$ and $f^{\prime}\left(x_{L}\right)=f^{\prime \prime}\left(x_{L}\right)=$ 
$f^{\prime}\left(x_{L}+3 h\right)=f^{\prime \prime}\left(x_{L}+3 h\right)=0$, then by solving equation (30) adapted for this case,

$$
\begin{aligned}
f(x)=A+\frac{B-A}{6 h^{3}}\left[\left(x-x_{L}\right)_{+}{ }^{3}-3\left(x-x_{L}-h\right)_{+}{ }^{3}\right. & \\
+ & \left.3\left(x-x_{L}-2 h\right)_{+}{ }^{3}-\left(x-x_{L}-3 h\right)_{+}{ }^{3}\right] .
\end{aligned}
$$

In the second case, if

$$
f\left(x_{L}\right)=f\left(x_{L}+3 h\right)=f^{\prime \prime}\left(x_{L}\right)=f^{\prime}\left(x_{L}+3 h\right)=f^{\prime \prime}\left(x_{L}+3 h\right)=0,
$$

but $f^{\prime}\left(x_{L}\right)=a$, then

$$
\begin{aligned}
f(x)=a\left(x-x_{L}\right)+\frac{a}{6 h^{2}}\left[-2\left(x-x_{L}\right)_{+}{ }^{3}+5\left(x-x_{L}-h\right)_{+}{ }^{3}\right. \\
\left.-4\left(x-x_{L}-2 h\right)_{+}{ }^{3}+\left(x-x_{L}-3 h\right)_{+}{ }^{3}\right] .
\end{aligned}
$$

In the third case, if $f\left(x_{L}\right)=f\left(x_{L}+3 h\right)=f^{\prime}\left(x_{L}\right)=f^{\prime \prime}\left(x_{L}\right)=f^{\prime \prime}\left(x_{L}+3 h\right)=0$, but $f^{\prime}\left(x_{L}+3 h\right)=b$, then

$$
\begin{aligned}
f(x)=\frac{b}{6 h^{2}}\left[-\left(x-x_{L}\right)_{+}{ }^{3}+\right. & 4\left(x-x_{L}-h\right)_{+}{ }^{3} \\
& \left.-5\left(x-x_{L}-2 h\right)_{+}{ }^{3}+2\left(x-x_{L}-3 h\right)_{+}{ }^{3}\right] .
\end{aligned}
$$

If $f\left(x_{L}\right)=A, f\left(x_{L}+3 h\right)=B, f^{\prime}\left(x_{L}\right)=a, f^{\prime}\left(x_{L}+3 h\right)=b, f^{\prime \prime}\left(x_{L}\right)=$ $f^{\prime \prime}\left(x_{L}+3 h\right)=0$, then $f(x)$ can be written as the sum of the three expressions (32), (33), and (34).

It is to be observed that for both the equidistant and the nonequidistant solutions of equations (30), the curved part is completely defined by the slopes and the end points of the given straight portions. The magnitudes of the ordinates at $x_{L+1}$ and $x_{L+2}$ do not enter into the computation; therefore, if fewer than two points are given in the gap between the end points of the two straight portions this method can also be applied. All that is necessary is to choose additional abscissas in the gap without fixing their ordinates.

A different situation prevails if the curved part between the two straight portions has more than two given points. Let the first straight line end at $x_{L}$ so that

$$
f\left(x_{L}\right)=A, \quad f^{\prime}\left(x_{L}\right)=a, \quad f^{\prime \prime}\left(x_{L}\right)=0
$$

and the next straight portion begin at $x_{M}$ so that

$$
f\left(x_{M}\right)=B, \quad f^{\prime}\left(x_{M}\right)=b, \quad f^{\prime \prime}\left(x_{M}\right)=0
$$

where $M-L>3$.

We can immediately give one such spline curve which satisfies at least all continuity requirements at its junctures with the two straight portions. This can be done by extending the first straight line until only three intervals have to be bridged by the curve. The following relations will have to be satisfied:

$$
\begin{array}{ll}
f_{0}\left(x_{M-3}\right)=A+a\left(x_{M-3}-x_{L}\right) & f_{0}\left(x_{M}\right)=B \\
f_{0}^{\prime}\left(x_{M-3}\right)=a & f_{0}^{\prime}\left(x_{M}\right)=b \\
f_{0}^{\prime \prime}\left(x_{M-3}\right)=0 & f_{0}^{\prime \prime}\left(x_{M}\right)=0 .
\end{array}
$$


The conditions at $x_{M-3}$ are immediately satisfied when we write

$$
\begin{aligned}
f_{0}(x)=A+a\left(x-x_{L}\right)+ & A_{M-3}\left(x-x_{M-3}\right)_{+}{ }^{3}+A_{M-2}\left(x-x_{M-2}\right)_{+}{ }^{3} \\
& +A_{M-1}\left(x-x_{M-1}\right)_{+}{ }^{3}+A_{M}\left(x-x_{M}\right)_{+}{ }^{3} .
\end{aligned}
$$

The coefficients $A_{M-3}, A_{M-2}, A_{M-1}$ follow from the conditions at $x_{M}$ whereas $A_{M}$ is a result of the requirement that the line should be straight for $x>x_{M}$.

Although such a spline curve satisfies the continuity requirements at $x_{L}$ and $x_{M}$, in general, it cannot be expected to pass closely enough to the interior points $\left(x_{L+1}, y_{L+1}\right),\left(x_{L+2}, y_{L+2}\right), \cdots,\left(x_{M-1}, y_{M-1}\right)$, nor is there any assurance that the curve will be free of fluctuations.

The particular solution of the problem of finding a spline curve for which equations (35) and (36) hold can be expanded into a general solution if we add linear combinations of spline curves which satisfy the conditions

$$
f\left(x_{L}\right)=f^{\prime}\left(x_{L}\right)=f^{\prime \prime}\left(x_{L}\right)=f\left(x_{M}\right)=f^{\prime}\left(x_{M}\right)=f^{\prime \prime}\left(x_{M}\right)=0 .
$$

The general solution satisfying (39) is of the form

$$
f(x)=\sum_{n=\mathrm{L}}^{M} A_{n}\left(x-x_{n}\right)_{+}^{3} \cdot
$$

The first three conditions of equations (39) are automatically satisfied. The last three conditions of equations (39) lead to three equations in $A_{L}, A_{L+1}, \cdots$, $A_{M-1}$, whereas $A_{M}$ is determined from the requirement that the spline curve be straight for $x>x_{M}$.

The three equations in $A_{L}, \cdots, A_{M-1}$ have coefficients which form a matrix of rank three, since it contains three rows which are, respectively, the third, second, and first powers of

$$
\left(x_{M}-x_{n}\right) \quad \text { for } n=L, L+1, \cdots, M-1 .
$$

Since the difference between the number of unknowns and the rank of a linear equation system gives the number of linearly independent solutions, we will have here $M-L-3$ linearly independent solutions.

One way of finding a special set of such independent solutions is to determine $M-L--3$ functions which satisfy the conditions

$$
f_{k}(x)=0
$$

$$
\begin{array}{r}
\text { for } x_{L} \leqq x \leqq x_{L+k-1} \\
\text { and for } x_{L+k+3} \leqq x \leqq x_{M}
\end{array}
$$

and which in the interval $x_{L+k-1}<x<x_{L+k+3}$ is controlled by the conditions

$$
\begin{array}{ll}
f_{k}{ }^{\prime}\left(x_{L+k-1}\right)=0 & f_{k}{ }^{\prime}\left(x_{L+k+3}\right)=0 \\
f_{k}^{\prime \prime}\left(x_{L+k-1}\right)=0 & f_{k}^{\prime \prime}\left(x_{L+k+3}\right)=0
\end{array}
$$

for $k=1,2, \cdots, M-L-3$.

These equations (41) and (42) are a case of equations (39) if we replace $L$ by $L+k-1$ and $M$ by $L+k+3$, and thus, $M-L$ becomes 4 . Therefore, for every value of $k=1,2, \cdots, M-L-3$, we get exactly one independent solution, $f_{k}(x)$. 
Since along with $f_{k}(x)$ any multiple of $f_{k}(x)$ is a solution of these equations, we normalize by requiring that

$$
f_{k}\left(x_{L+k}\right)=1 .
$$

These functions $f_{k}(x)$ are then shown to be linearly independent in the following way.

If there were to exist a relation

$$
\sum_{k=1}^{M-\mathbf{L}-3} V_{k} f_{k}(x)=0
$$

then substituting $x=x_{L+1}$ would yield $V_{1}=0$, and, successively, all $V_{\dot{x}}$ could be shown to be zero.

The general solution of equations (35) and (36) is then found by adding to (37) the general solution of (39), or what amounts to the same, a linear combination of solutions of (41) and (42).

We thus obtain

$$
f(x)=f_{0}(x)+\sum_{k=1}^{M-\mathrm{L}-3} p_{k} f_{k}(x) .
$$

The coefficients $p_{k}$ are to be determined in such a way that

$$
\sum_{n=\mathrm{L}+1}^{M-1}\left[f\left(x_{n}\right)-y_{n}\right]^{2}+s \sum_{n=\mathrm{L}+1}^{M-1}\left[f^{\prime \prime}\left(x_{n}\right)-r_{n}\right]^{2}
$$

becomes a minimum.

The larger the value of $s$ the more emphasis is placed on the agreement of the second derivative $f^{\prime \prime}\left(x_{n}\right)$ of the curve with the second differences $r_{n}$ of the given data. It is to be observed that in this case we have $M-L-3$ coefficients, and we want to have agreement between the second derivatives and the second differences at $M-L-1$ points, so that no matter how large an $s$ is taken we can only minimize the discrepancies but cannot force the discrepancies to zero. In most cases we will easily achieve agreement of sign between the second derivatives and the second differences with a suitable value of $s$.

Should a case arise where it cannot be achieved, we can take recourse to the following procedure. Agreement in sign between the second derivative and second difference can be forced if we permit some of the quantities to vary that have been held constant previously. Such quantities are, for instance, the constants which characterize the straight portions; namely, the quantities $a, A, b, B$, of equations (35) and (36).

It may be of interest to note that in the case of equidistant abscissas $x_{n}$, the solution of equations (41) and (42) can be given explicitly in comparatively simple form.

If $x_{L+k}=x_{L}+k h$, then a simple verification shows that

$$
\begin{aligned}
g(x)=\left(x-x_{L}\right)^{3}-4(x & \left.-x_{L}-h\right)_{+}{ }^{3}+6\left(x-x_{L}-2 h\right)_{+}{ }^{3} \\
& -4\left(x-x_{L}-3 h\right)_{+}{ }^{3}+\left(x-x_{L}-4 h\right)_{+}{ }^{3}
\end{aligned}
$$


can be taken as $f_{1}(x)$, and all the $f_{k}(x)$ are found by the formula

$$
f_{k}(x)=g[x-(k-1) h] .
$$

Thus, we have developed a procedure for finding spline curves into which we incorporate straight portions. The methods described apply to abscissas that are not necessarily equally spaced, but they simplify considerably in the case of equidistant abscissas.

5. Applications. The various procedures described so far were applied to both realistic and artificially created data. We now want to describe in some detail two particular problems which were solved on the IBM 704.

In the first case, a set of faired lines for DD 948-949 were available. A small number of offsets were selected as the basis of a problem. We applied our method to them as if they were taken as part of a preliminary design, and reconstructed from them a large number of points in order to compare them with the corresponding faired data.

In particular, eighteen points on each of five waterlines were chosen. The chosen waterlines were $2 \mathrm{ft}, 4 \mathrm{ft}, 6 \mathrm{ft}, 10 \mathrm{ft}$, and $14 \frac{1}{2} \mathrm{ft}$. The point $\left(x_{0}, y_{0}\right)$ on each waterline was the tabulated initial point of that line so that $x_{0}$ was not the same for all waterlines. The other abscissas $x_{1}, \cdots, x_{17}$ were chosen so that $x_{n}=20 n \mathrm{ft}$.

With these 90 given points, the coefficients of the function representing the major part of the ship's underwater surface were determined by the method of Section 3.

This permitted us to compute any number of offsets and, in particular, we were able to tabulate the offsets at 2 - $\mathrm{ft}$ spacing on each of the input waterlines and also on the 8 -ft and 12 -ft waterlines.

These computed offsets were then compared with the given faired offsets and the discrepancies were found to be no greater than $\frac{5}{8}$-in. at any point.

This example, used as a practice case, was based on input points which were already well faired; therefore, a small value of $s$, namely, $s=1$, in formula (21) gave no fluctuations. Also, when the interpolation method described in Section 2 was applied to the same data no fluctuations appeared.

A practical application of the methods described here was made in the solution of a problem proposed by the New York Naval Shipyard.

It was requested that faired ship lines for LPD 1 be computed. A preliminary design was given, including a table which contained the offsets on each of 11 waterlines for stations that were generally $25 \mathrm{ft}$ apart. The problem was to compute the offsets for each frame at 2 -ft spacing.

A study of the preliminary design of the ship showed a feature which required some extension of the method described previously. Thus far we have considered only ships whose lines have continuous slope and curvature at every point. This ship, the LPD 1, has a horizontal knuckle $22 \mathrm{ft}$ above the keel extending from the midsection to the after perpendicular. At this knuckle the section lines have a discontinuity of the first derivative.

The waterlines can be handled here in the same way as previously described. If we were to continue as in the case where there is no knuckle and form a surface $y=F(x, z)$, we would be led either to violent fluctuations or, if $s$ in formula (21) 
TABLE 1

Waterline Half-Breadths Furnished by New York Naval Shipyard for $L P D 1$

(From Station 18, $x=450 \mathrm{ft}$, to Aft Perpendicular, $x=500 \mathrm{ft}$; Station Spacing $25 \mathrm{ft}$. Dimensions in $\mathrm{ft}$-in.-8ths)

\begin{tabular}{c|c|c|c|c}
\hline Station & 22-Ft W.L. & 28-Ft W.L. & 36-FtW.L. & 44-Ft W.L. \\
\cline { 2 - 3 } \cline { 4 - 4 } 18 & $35-1-4$ & $35-11-4$ & $37-0-6$ & $38-2-0$ \\
19 & $31-11-4$ & $32-11-6$ & $34-4-2$ & $35-9-0$ \\
AP & $28-4-0$ & $29-7-0$ & $31-3-4$ & $33-0-0$ \\
\hline
\end{tabular}

were chosen sufficiently large, the knuckle would be completely faired out, which is not what was desired.

We can avoid this difficulty by subdividing the coefficients of the various waterlines into two groups: those that go with the fore part of the ship where there is no knuckle, and those that go with the aft part of the ship where there is a knuckle. The fore part is treated in the usual manner. In the aft part, we fit the coefficients of the waterlines above the knuckle separately and also the coefficients of the waterlines below the knuckle separately.

In the actual computation, formula (21) was used with $s=1$, which gave satisfactory results. The result of the computation was printed by the high-speed printer associated with the computing machine. It was arranged in the form of tables giving offsets on every frame. The frames were spaced $2 \mathrm{ft}$ apart, and on each frame the offsets were given for every waterline level $2 \mathrm{ft}$ apart.

As an illustration, a small part of the input data furnished by the New York Naval Shipyard is presented in Table 1, and the corresponding output computed by the IBM 704 is given in Table 2 .

Table 1 contains the portions above the knuckle of Stations 18, 19, and the After Perpendicular.

The computed data for the same region are given in Table 2 for Frames 225 to After Perpendicular (Frame 250), which corresponds to a length of $50 \mathrm{ft}$, and for the $22-\mathrm{ft}$ to $44-\mathrm{ft}$ waterlines.

Tables 1 and 2 contain what is essentially $1 / 20$ of the actual input and output data.

6. Conclusion. The methods derived here were developed to serve as a means for finding an arbitrarily large number of full-scale offsets if only a limited number of offsets, taken from a preliminary design, are given. This particular use guided us in the choice of the type of curve to represent a typical ship line. As was made clear, particularly in Section 3, special care was taken to avoid unwanted fluctuations.

If we fit a whole waterline or a half waterline with a single polynomial, we do not have any assurance that our lines are free from unwanted fluctuations. Even a least-squares procedure will not necessarily exclude unwanted fluctuations, since it controls only the discrepancies between given and computed points and does not put any constraint on the curvature. In Section 3 we described a method where such a constraint on the curvature is applied. 
TABLE 2

Waterline Half-Breadths Computed on IBM 704 for LPD 1

(From Frame 225, $x=450 \mathrm{ft}$, to Frame 250, $x=500 \mathrm{ft}$; Frame Spacing $2 \mathrm{ft}$. Dimensions in $\mathrm{ft}$-in.-8ths)

\begin{tabular}{|c|c|c|c|c|c|c|}
\hline Frame & 22-Ft W.L. & 24-Ft W.L. & 26-Ft W.L. & 28-Ft W.L. & 30-Ft W.L. & 32-Ft W.L. \\
\hline $\begin{array}{l}225 \\
226 \\
227 \\
228 \\
229 \\
230 \\
231 \\
232 \\
233 \\
234 \\
235 \\
236 \\
237 \\
238 \\
239 \\
240 \\
241 \\
242 \\
243 \\
244 \\
245 \\
246 \\
247 \\
248 \\
249 \\
250\end{array}$ & $\begin{array}{l}35-1-4 \\
34-10-5+* \\
34-7-6+ \\
34-4-7 \\
34-2-0-* \\
33-11-0- \\
33-8-0- \\
33-4-7+ \\
33-1-7- \\
32-10-6- \\
32-7-4+ \\
32-4-3- \\
32-1-1 \\
31-9-7 \\
31-6-5- \\
31-3-2 \\
30-11-7 \\
30-8-4- \\
30-5-0+ \\
30-1-5- \\
29-10-1- \\
29-6-4+ \\
29-3-0- \\
28-11-3- \\
28-7-5+ \\
28-4-0\end{array}$ & $\begin{array}{l}35-4-7- \\
35-2-1- \\
34-11-2 \\
34-8-3+ \\
34-5-4+ \\
34-2-5 \\
33-11-5+ \\
33-8-5+ \\
33-5-5 \\
33-2-4+ \\
32-11-4- \\
32-8-3- \\
32-5-1+ \\
32-2-0- \\
31-10-6- \\
31-7-4- \\
31-4-1+ \\
31-0-7- \\
30-9-4- \\
30-6-1- \\
30-2-5 \\
29-11-1+ \\
29-7-5+ \\
29-4-1 \\
29-0-4+ \\
28-8-7+\end{array}$ & $\begin{array}{l}35-8-1+ \\
35-5-4- \\
35-2-6- \\
34-11-7+ \\
34-9-1- \\
34-6-2 \\
34-3-3- \\
34-0-3+ \\
33-9-3+ \\
33-6-3+ \\
33-3-3 \\
33-0-2+ \\
32-9-2- \\
32-6-1- \\
32-2-7+ \\
31-11-6- \\
31-8-4- \\
31-5-2- \\
31-1-7+ \\
30-10-5- \\
30-7-2- \\
30-3-7- \\
30-0-3+ \\
29-9-0- \\
29-5-4- \\
29-1-7+\end{array}$ & $\begin{array}{l}35-11-4 \\
35-8-7- \\
35-6-1+ \\
35-3-3+ \\
35-0-5+ \\
34-9-7 \\
34-7-0 \\
34-4-1 \\
34-1-2 \\
33-10-2+ \\
33-7-3- \\
33-4-2+ \\
33-1-2 \\
32-10-2- \\
32-7-1- \\
32-4-0- \\
32-0-6+ \\
31-9-5 \\
31-6-3 \\
31-3-1 \\
30-11-7 \\
30-8-4+ \\
30-5-2- \\
30-1-7- \\
29-10-3+ \\
29-7-0\end{array}$ & $\begin{array}{l}36-2-6+ \\
36-0-2- \\
35-9-5- \\
35-6-7+ \\
35-4-2- \\
35-1-4 \\
34-10-6- \\
34-7-7 \\
34-5-0+ \\
34-2-1+ \\
33-11-2 \\
33-8-2+ \\
33-5-3- \\
33-2-3- \\
32-11-2+ \\
32-8-2 \\
32-5-1+ \\
32-2-0+ \\
31-10-7 \\
31-7-6- \\
31-4-4 \\
31-1-2+ \\
30-10-0+ \\
30-6-6 \\
30-3-3+ \\
30-0-1-\end{array}$ & $\begin{array}{l}36-6-1 \\
36-3-5- \\
36-1-0+ \\
35-10-3+ \\
35-7-6+ \\
35-5-1 \\
35-2-3 \\
34-11-5 \\
34-8-7 \\
34-6-0+ \\
34-3-2- \\
34-0-3- \\
33-9-3+ \\
33-6-4 \\
33-3-4+ \\
33-0-4+ \\
32-9-4 \\
32-6-4- \\
32-3-3+ \\
32-0-3- \\
31-9-2- \\
31-6-0+ \\
31-2-7 \\
30-11-5+ \\
30-8-4- \\
30-5-2-\end{array}$ \\
\hline Frame & 34-Ft W.L. & 36-Ft W.L. & 38-Ft W.L. & 40-FtW.L. & 42-FtW.L. & 44-Ft W.L. \\
\hline $\begin{array}{l}225 \\
226 \\
227 \\
228 \\
229 \\
230 \\
231 \\
232 \\
233 \\
234 \\
235 \\
236 \\
237 \\
238 \\
239 \\
240 \\
241 \\
242 \\
243 \\
244 \\
245 \\
246 \\
247 \\
248 \\
249 \\
250\end{array}$ & $\begin{array}{l}36-9-3+ \\
36-7-0- \\
36-4-4- \\
36-1-7+ \\
35-11-3- \\
35-8-6 \\
35-6-1- \\
35-3-3+ \\
35-0-5+ \\
34-9-7+ \\
34-7-1+ \\
34-4-3- \\
34-1-4 \\
33-10-5+ \\
33-7-6 \\
33-4-7- \\
33-1-7+ \\
32-11-0- \\
32-8-0- \\
32-4-7+ \\
32-1-7 \\
31-10-7- \\
31-7-6 \\
31-4-5 \\
31-1-4 \\
30-10-3-\end{array}$ & $\begin{array}{l}37-0-6 \\
36-10-3- \\
36-7-7+ \\
36-5-3+ \\
36-2-7+ \\
36-0-3 \\
35-9-6+ \\
35-7-1+ \\
35-4-4 \\
35-1-7- \\
34-11-1 \\
34-8-3 \\
34-5-5 \\
34-2-7- \\
34-0-0 \\
33-9-1+ \\
33-6-2+ \\
33-3-3+ \\
33-0-4 \\
32-9-5- \\
32-6-5 \\
32-3-5 \\
32-0-5 \\
31-9-5- \\
31-6-4+ \\
31-3-4-\end{array}$ & $\begin{array}{l}37-4-0+ \\
37-1-6- \\
36-11-3- \\
36-8-7+ \\
36-6-4 \\
36-4-0 \\
36-1-4 \\
35-10-7+ \\
35-8-3 \\
35-5-6 \\
35-3-1 \\
35-0-3+ \\
34-9-6 \\
34-7-0+ \\
34-4-2+ \\
34-1-4 \\
33-10-6 \\
33-7-7+ \\
33-5-1- \\
33-2-2- \\
32-11-3- \\
32-8-4- \\
32-5-4 \\
32-2-5- \\
31-11-5 \\
31-8-5\end{array}$ & $\begin{array}{l}37-7-3- \\
37-5-1- \\
37-2-6+ \\
37-0-4- \\
36-10-0+ \\
36-7-5+ \\
36-5-2- \\
36-2-6- \\
36-0-2- \\
35-9-5+ \\
35-7-1- \\
35-4-4 \\
35-1-7 \\
34-11-2 \\
34-8-5- \\
34-5-7 \\
34-3-1+ \\
34-0-3+ \\
33-9-5+ \\
33-6-7 \\
33-4-1- \\
33-1-2 \\
32-10-3+ \\
32-7-4+ \\
32-4-5+ \\
32-1-6\end{array}$ & $\begin{array}{l}37-10-5+ \\
37-8-4- \\
37-6-2 \\
37-4-0- \\
37-1-5+ \\
36-11-2+ \\
36-8-7+ \\
36-6-4 \\
36-4-1- \\
36-1-5 \\
35-11-1 \\
35-8-5- \\
35-6-0+ \\
35-3-4- \\
35-0-7 \\
34-10-2 \\
34-7-5 \\
34-5-0- \\
34-2-2+ \\
33-11-5- \\
33-8-7- \\
33-6-1- \\
33-3-3- \\
33-0-4+ \\
32-9-6- \\
32-6-7\end{array}$ & $\begin{array}{l}38-2-0 \\
37-11-7- \\
37-9-5+ \\
37-7-4 \\
37-5-2 \\
37-3-0- \\
37-0-5+ \\
36-10-3- \\
36-8-0- \\
36-5-5- \\
36-3-1+ \\
36-0-6- \\
35-10-2- \\
35-7-6- \\
35-5-2- \\
35-2-5+ \\
35-0-1- \\
34-9-4 \\
34-6-7+ \\
34-4-2 \\
34-1-5 \\
33-10-7+ \\
33-8-2- \\
33-5-4 \\
33-2-6 \\
33-0-0-\end{array}$ \\
\hline
\end{tabular}

* Plus sign indicates $+\frac{1}{24}$ in.; minus sign indicates $-\frac{1}{24}$ in. 
The representation of a single line by means of different cubics for different parts of the line, which might be cumbersome for hand calculations, does not constitute any difficulty for a high-speed computer.

\section{Applied Mathematics Laboratory David Taylor Model Basin \\ Washington 7, D. C.}

1. D. W. TAYLOR, "Calculations for ships' forms and the light thrown by model experiments upon resistance, propulsion, and rolling of ships," Trans. International Engrg. Congress, San Francisco, 1915.

2. H. E. SAUNDER, Hydrodynamics in Ship Design, v. 2, The Society of Naval Architects and Marine Engineers, New York, 1957, p. 186-205.

3. W. H. Rösingh \& J. Berghius, "Mathematical ship form," International Shipbuilding Progress, v. 6, January 1959.

4. P. C. PIEn, Mathematical Ship Surface, David Taylor Model Basin Report 1398, January 1960 .

5. J. E. KERwiN, "Polynomial surface representation of arbitrary ship forms," $J$. of Ship Research, v. 4, June 1960. 